\title{
Tratamento de Resíduos de Serviços de Saúde pelo processo de pirólise
}

\author{
Health waste treatment by pyrolysis \\ Artur Tôrres Filho', Alison Frederico Medeiros Ferreira², \\ Gilberto Caldeira Bandeira de Melo $^{3}$, Liséte Celina Lange ${ }^{4}$
}

口-

\section{RESUMO}

O presente trabalho apresenta a avaliação técnica e ambiental de uma planta piloto de tratamento de Resíduos de Serviços de Saúde (RSS) por pirólise quanto à produtividade, ao consumo de combustível, ao rendimento gravimétrico do processo, às emissões atmosféricas, aos efluentes líquidos e ao produto carbonizado obtido. O processo apresentou valores para perda de massa entre 46,75 e 58,77\%, para tempos de processamento variando entre 2 e 6 horas. A tecnologia empregada demonstrou maior segurança ambiental na terceira condição de operação testada, em relação às emissões de contaminantes para a atmosfera, levando-se em consideração o valor obtido na concentração de dioxinas e furanos de 0,0162 ng/ $\mathrm{Nm}^{3}$. Nessa condição, a operação ocorreu com dois combustores, gás de pirólise sendo injetado no duto de alimentação de biomassa e com incremento de 15\% no volume do fluxo de ar na zona de combustão dos cavacos, em mistura com o gás de pirólise. A solução proposta apresentou potencial para ser aplicada em municípios que enfrentam o gerenciamento adequado dos RSS como um desafio a ser transposto.

Palavras-chave: pirólise; tratamento de resíduos; resíduos de serviços de saúde; biochar.

\begin{abstract}
This work shows the technical and environmental evaluation of a pilot plant for treatment of health waste by pyrolysis, regarding its productivity, fuel consumption, weight loss in the process, atmospheric emissions, wastewater and the charcoal obtained The process resulted in values of weight loss between 46,75 and 58,77\%, for processing time ranged between 2 and 6 hours The greatest environmental safety was obtained in the third tested operation condition, concerning atmospheric emissions of pollutants, as the concentration of dioxins and furans value obtained was $0,0162 \mathrm{ng} / \mathrm{Nm}^{3}$. Two combustors were used in the operation, pyrolysis gas was injected in the biomass feed pipe, and the air flow volume was increased by $15 \%$ in the combustion zone of the wood chips, mixed with the pyrolysis gas. The proposed solution has shown great potential to be used in municipalities dealing with proper management of health waste as a challenge to be overcome
\end{abstract}

Keywords: pyrolysis; waste treatment; health waste; biochar.

\section{INTRODUÇÃO}

Os resíduos de serviços de saúde (RSS) aparecem em evidência como um dos grandes desafios da atualidade, pois seu tratamento e disposição final são submetidos a uma regulação específica no Brasil, através da resolução no 358/2005 do Conselho Nacional de Meio Ambiente. Os RSS são gerados em diferentes estabelecimentos que prestam serviços de saúde, tais como hospitais, clínicas médicas e veterinárias, laboratórios de análises clínicas, farmácias e unidades básicas de saúde. Dos 5.564 municípios brasileiros, $80 \%$ possuem coleta dos RSS, perfazendo um total de aproximadamente 8.909 toneladas coletadas, por dia, no país (PNSB, 2008).

O município de Belo Horizonte, com mais de dois milhões de habitantes, concentra o maior número de estabelecimentos de prestação de serviços de saúde do estado de Minas Gerais, para onde converge grande parte da população do estado, para os mais diversos tipos de tratamento de saúde. Os estabelecimentos de saúde, administrados pela prefeitura

'Doutorando em Saneamento, Meio Ambiente e Recursos Hídricos pela Universidade Federal de Minas Gerais (UFMG) - Belo Horizonte (MG), Brasil. Diretor técnico da Engenho Nove Engenharia Ambiental Ltda.

${ }^{2}$ Mestre em Saneamento, Meio Ambiente e Recursos Hídricos pela UFMG - Belo Horizonte (MG), Brasil.

${ }^{3}$ Pós Doutor pela Technische Universitat Hamburg-Harburg (TUHH) - Hamburg, Alemanha. Professor associado ao Departamento de Engenharia Sanitária e Ambiental da UFMG - Belo Horizonte (MG), Brasil.

${ }^{4}$ Doutora em Tecnologia Ambiental pela Universidade de Londres - Londres, Inglaterra. Professora associada ao Departamento de Engenharia Sanitária e Ambiental da UFMG - Belo Horizonte (MG), Brasil.

Endereço para correspondência: Artur Tôrres Filho - Rua universo, 245 - Santa Lúcia - 30350-612 - Belo Horizonte (MG), Brasil - E-mail: artur@engenho9.com.br

Recebido: 14/11/12 - Aceito: 27/11/13 - Reg. ABES: 717 
municipal, encaminham desde o início dos anos 1980 cerca de 40 toneladas de RSS geradas diariamente nas unidades municipais de saúde, para aterramento na Central de Tratamento de Resíduos Sólidos (CTRS) do município de Belo Horizonte, com os resíduos sendo dispostos atualmente em uma vala séptica. A CTRS encerrou as atividades de aterramento de resíduos sólidos urbanos em 2005. Atualmente, operam no local, além da vala séptica de RSS, uma unidade de compostagem e uma estação de transbordo de resíduos sólidos urbanos (RSU) para outro aterro sanitário.

Diante da demanda por uma solução para o tratamento adequado dos RSS gerados pela rede municipal de saúde, foi firmado um convênio tecnológico entre o poder público municipal, a Universidade Federal de Minas Gerais e uma instituição privada, para a avaliação da viabilidade técnica e ambiental da aplicação do processo de pirólise para tratamento térmico dos resíduos. A tecnologia selecionada apresentou uma vantagem competitiva em relação a outros processos térmicos, tais como incineração e plasma,uma vez que deveria utilizar, como combustível para o processo, cavacos de madeira a serem produzidos na própria CTRS, com os resíduos de podas de árvores e supressão vegetal do município. Com mais de $80 \mathrm{~m}^{2}$ de área verde por habitante, o município de Belo Horizonte encaminha mensalmente para a CTRS mais de 1.000 toneladas de material lenhoso provenientes das podas e supressão de árvores. Esse material lenhoso, em função da desuniformidade nas suas dimensões e do alto teor de umidade, não possui valor econômico, sendo destinado às operações de aterramento.

Atualmente no país, segundo a Resolução CONAMA 358/2005 e ANVISA RDC 306/2004 os resíduos de serviços de saúde são classificados em cinco grupos, conforme a Tabela 1.

Existem várias tecnologias no mundo aplicáveis ao tratamento de RSS, que podem ser classificadas nas seguintes categorias (USEPA, 2011):

- Térmica: aquecimento seco ou úmido, microondas, infravermelho, laser, plasma e pirólise;

- Química: cloro e derivados de cloro, ozônio e enzimas;

- Radiação: ultravioleta e cobalto-60.

Todas as tecnologias apresentadas possuem suas vantagens e desvantagens, com vários níveis de segurança, custos e impactos sobre o meio ambiente. Contudo, segundo Melo et al. (2008), os tratamentos como a incineração e plasma, apesar de ocasionarem uma redução acima de $90 \%$ em massa e volume do resíduo, possuem algumas desvantagens que podem inviabilizar a sua implantação, como por exemplo,

Tabela 1 - Classificação dos Resíduos de Serviços de Saúde na resolução CONAMA nº 358/05 e RDC n. ${ }^{\circ}$ 306/04.

\begin{tabular}{l|c}
\hline Grupo & Características \\
A & Biológico \\
\hline B & Químico \\
\hline C & Radioativo \\
\hline D & Semelhante aos Resíduos Sólidos Urbanos \\
\hline E & Perfurantes, cortantes e abrasivos. \\
\hline
\end{tabular}

alto custo de implantação inicial, de manutenção e operação além da necessidade de mão-de-obra especializada.

Huffman e Lee (1996) apontam a incineração como a tecnologia mais comumente utilizada nos Estados Unidos para tratamento de RSS, responsável por tratar mais de 80\% dos RSS gerados no país. Os mesmos autores indicam as dioxinas e os furanos como os grupos de substâncias que consistem na maior preocupação em relação às emissões de gases para a atmosfera nesse processo de tratamento. Na mesma linha, Alvim-Ferraz e Afonso (2003) confirmaram uma forte influência da segregação e mistura dos diferentes tipos de RSS na composição das emissões gasosas de um incinerador, bem como da necessidade de injeção de combustível auxiliar para o controle adequado da combustão, em função do baixo poder calorífico dos RSS.

Diante do cenário atual e da dificuldade de equalização dos custos, riscos ambientais e redução de massa/volume dos resíduos, a pirólise apresenta-se como uma opção promissora no tratamento de RSS. A pirólise caracteriza-se pela degradação térmica dos resíduos em uma atmosfera com deficiência de oxigênio, minimizando, portanto, as emissões de poluentes formados em atmosfera oxidante, tais como as dioxinas e os furanos. Porém, uma dificuldade encontrada na pirólise é o fato de que, para a quebra de polímeros no processo de termoconversão, é necessária uma considerável quantidade de energia para quebra das macromoléculas, sendo desejável a utilização da energia gerada no processo para compensar total ou parcialmente os custos energéticos (BRANDRUP, 1996). Nos últimos 20 anos, muitos estudos sobre o processo de pirólise têm sido publicados, relatando a minimização das emissões de $\mathrm{NO}_{\mathrm{x}^{\prime}}, \mathrm{SO}_{2}$ e metais pesados em relação ao processo de incineração (AVENELL et al., 1996). Malkow (2004) apresenta um estudo com dez tecnologias de pirólise e gaseificação para o tratamento de RSU, concluindo que os processos de pirólise e gaseificação podem aumentar a eficiência energética através da geração de energia elétrica e a utilização dos subprodutos, possibilitando a redução das emissões de compostos perigosos. Deng et al. (2003) fizeram análise termogravimétrica em 14 diferentes tipos de RSU, em um reator de pirólise, variando a temperatura entre 300 e $1000^{\circ} \mathrm{C}$, concluindo que as maiores perdas de massa ocorrem entre 310 e $450^{\circ} \mathrm{C}$. Zhu et al. (2008) desenvolveram um estudo de pirólise de compostos típicos de RSU, utilizando a análise termogravimétrica em temperaturas de até $960^{\circ} \mathrm{C}$. De forma similar aos outros autores, observaram que as maiores perdas de massa ocorreram na faixa de temperatura compreendida entre 313 e $494^{\circ} \mathrm{C}$.

Caballero et al. (1998) realizaram estudos sobre a cinética das reações que envolvem a decomposição térmica de resíduos submetidos a pirólise, com diferentes proporções de oxigênio na atmosfera do reator. A introdução do oxigênio no processo proporcionou o surgimento de reações de oxidação e combustão do material carbonizado, reações fundamentais para a redução mássica da fase sólida. O modelo proposto por Caballero et al. (1998) apresenta a Equação 1 como equação geral para o processo de pirólise. 
$B \stackrel{k}{\rightarrow} r C+(1-r) V$

Na qual:

$\mathrm{B}=$ matéria orgânica total $(\mathrm{kg})$;

$\mathrm{C}=$ produto carbonizado formado $(\mathrm{kg})$;

$\mathrm{V}=$ voláteis $(\mathrm{kg})$;

$\mathrm{r}=$ coeficiente de rendimento $(\mathrm{kg}$ de carvão formado/ $\mathrm{kg}$ de matéria orgânica decomposta);

$\mathrm{k}=$ Constante cinética.

Debela et al. (2012) investigaram a aplicação do produto obtido através da pirólise de biomassa a baixa temperatura $\left(200 \mathrm{e} 400^{\circ} \mathrm{C}\right)$ para tratamento de solos contaminados por metais pesados, afirmando que o biochar produzido possui potencial para remediação de solos, visando a imobilização de metais pesados. Beesley et al. (2011) descrevem em seus estudos o potencial de aplicação de biochar para remediação de solos, alertando, no entanto, para a importância de se estabelecer taxas de aplicação adequadas para esse produto. Smith et al. (2010) investigaram as diferentes taxas de emissão de $\mathrm{CO}_{2}$ no solo, após aplicação de biochar como condicionador. A aplicação do produto carbonizado obtido a partir do processo de pirólise (biochar) em solos, tem sido, portanto, uma alternativa de disposição estudada por diversos autores, apesar da vocação energética para utilização como combustível, diante do considerável poder calorífico desse produto.

O presente trabalho apresenta a avaliação técnica e ambiental de uma tecnologia proposta para o tratamento térmico pelo processo de pirólise dos RSS gerados pelos estabelecimentos da rede pública municipal de saúde do município de Belo Horizonte, quanto à produtividade, ao consumo de combustível, ao rendimento gravimétrico do processo, às emissões atmosféricas, aos efluentes líquidos e ao produto carbonizado obtido.

\section{METODOLOGIA}

Este trabalho insere-se no Convênio de Cooperação Técnica, intitulado "Avaliação Tecnológica de Processo Térmico para Tratamento de Resíduos de Serviços de Saúde”. O aparato experimental foi operado na CTRS do município de Belo Horizonte. Um aparato experimental em escala plena, com capacidade de processamento de $3.000 \mathrm{~L}$ de RSS por ciclo, foi implantado e operado no período de janeiro a março de 2012. O processo de tratamento térmico de RSS por pirólise é ilustrado na Figura 1. $\mathrm{O}$ aparato experimental montado consiste de um dispositivo tecnológico denominado "Pyrolix", com depósito de patente junto ao INPI sob o número 14090002634, e titularidade pertencente à Universidade Federal de Minas Gerais (UFMG) e a instituições co-titulares.

O processo de pirólise utilizado caracteriza-se pelo uso de um fluido térmico circulante para o aquecimento do reator de pirólise, do qual são encaminhados os gases para a fornalha, que promove o aquecimento do referido fluido. Os resíduos gerados nas unidades municipais de saúde, pertencentes ao grupo A, foram recebidos e estocados em câmara fria, em uma faixa de temperatura de 0 a $2^{\circ} \mathrm{C}$, sendo fornecidos aleatoriamente para o experimento. Antes do carregamento do reator, os resíduos foram pesados, visando o dimensionamento da carga de processo e a verificação da perda de massa ocorrida após o tratamento.

Os resíduos para tratamento foram encaminhados pela esteira de alimentação até o reator (3), iniciando, a partir desta fase, o processo de tratamento térmico propriamente dito. Após cada carregamento, o reator foi submetido a aquecimento indireto, por meio de fluido térmico, o que promoveu dessa forma a carbonização do RSS, com o fluido sendo mantido em temperaturas entre 315 e $330^{\circ} \mathrm{C}$, e o sistema de pós-queima operando a uma temperatura em torno de $750^{\circ} \mathrm{C}$. Combustores de biomassa (7) foram instalados, de forma a injetar o combustível auxiliar na fornalha (8), para manutenção das temperaturas de processo. O processo ocorreu em ciclos intermitentes (bateladas). O sistema contou com um tratamento de gases por via úmida (9), antes do lançamento final para a atmosfera. A solução de lavagem de gases foi mantida em uma faixa de $\mathrm{pH}$ entre 9 e 10, através da adição de $\mathrm{NaOH}$, em circuito fechado, com reposição do líquido evaporado, em um reservatório (10) de 30.000 L. O aparato experimental era dotado de um sistema automatizado para manutenção das temperaturas de processo e alimentação de combustível auxiliar, através de controlador lógico programável.

As avaliações ocorreram em 14 ciclos completos de operação. Foram realizados ciclos para avaliação dos tempos de processo, relacionando-os à perda de massa ocorrida no material após o processamento e o consumo específico de combustível em cada ciclo. A operação iniciava-se com o aquecimento do fluido térmico até a temperatura de $315^{\circ} \mathrm{C}$, com o calor sendo obtido exclusivamente pela combustão dos cavacos de madeira. Após o fluido atingir esta temperatura, o reator era carregado com RSS. Para avaliação das emissões atmosféricas da planta, foram testadas três diferentes condições de operação:

- Condição 1: Operação com um combustor de biomassa (cavacos de madeira), com o gás de pirólise sendo injetado diretamente na fornalha de pós queima;

- Condição 2: Operação com dois combustores, com o gás de pirólise sendo injetado junto com a biomassa;

- Condição 3: Operação com dois combustores, com o gás de pirólise sendo injetado junto com a biomassa, com incremento de $15 \%$ no volume do fluxo de ar na zona de combustão dos cavacos, em mistura com o gás de pirólise.

Ao final de cada ciclo de processo, o resfriamento do resíduo carbonizado ocorreu no próprio reator, proporcionado através do desvio da linha de recirculação do fluido térmico para o circuito alternativo de resfriamento. O produto obtido apresentou-se na forma de material orgânico carbonizado, em mistura com alguns elementos metálicos. 


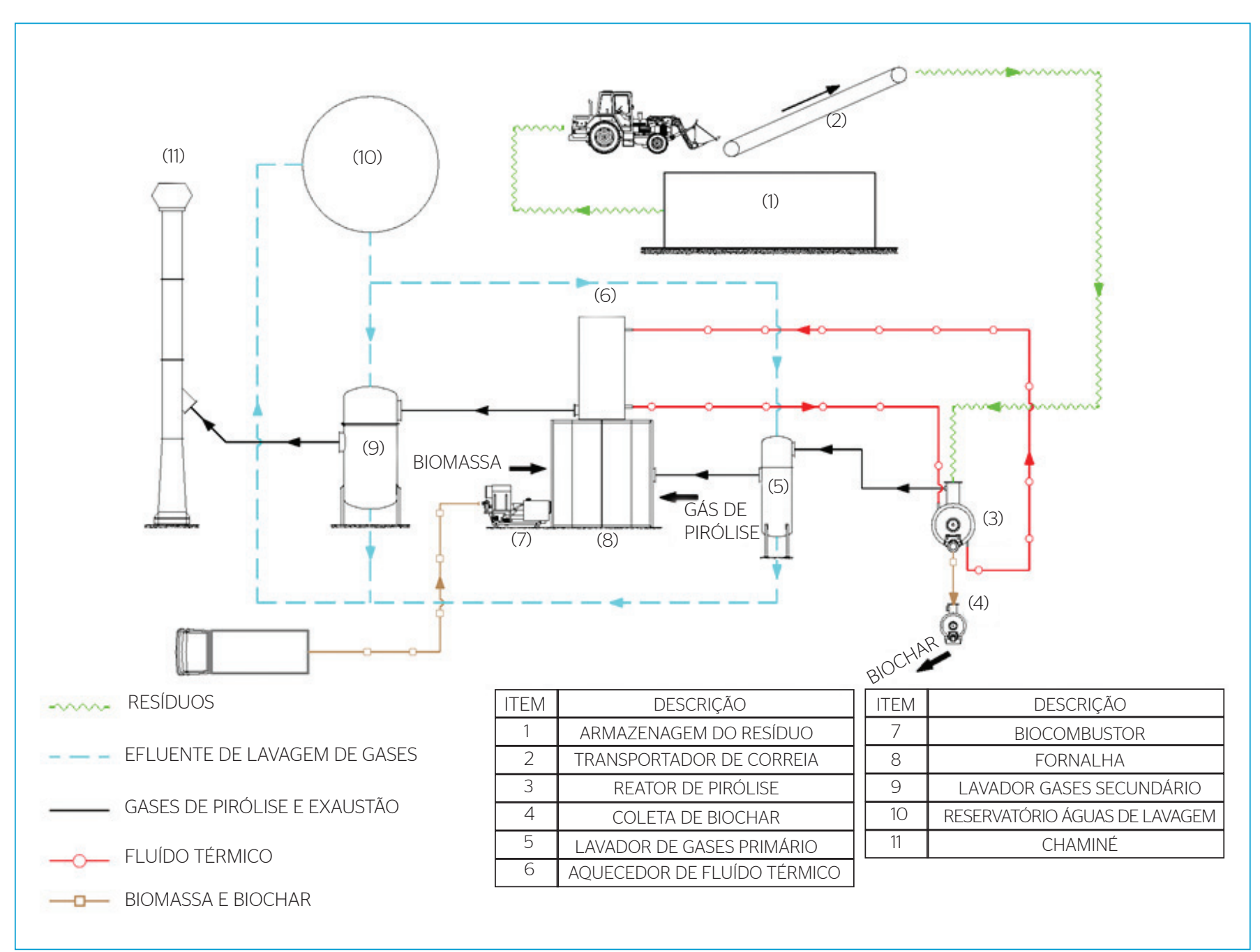

Figura 1 - Fluxograma do processo de pirólise.

$\mathrm{Na}$ avaliação das emissões atmosféricas, contemplou-se nas três condições de operação, a medição da vazão, as concentrações de material particulado (MP), $\mathrm{SO}_{2}, \mathrm{HCl}, \mathrm{HBr}, \mathrm{HF}$, fluoretos, gás cloro, dioxinas e furanos, óxidos de nitrogênio $\left(\mathrm{NO}_{\mathrm{x}}\right), \mathrm{CO}$ e metais $(\mathrm{Mn}, \mathrm{Cu}, \mathrm{Pb}, \mathrm{Cd}, \mathrm{Hg}, \mathrm{Ti}, \mathrm{As}, \mathrm{Co}$, $\mathrm{Ni}$, Se, Te, Sb, Cr, Pt, Pd, Rh, V e Sn), assim como o cálculo da vazão normalizada. Foram aplicados testes de hipóteses para a avaliação estatística dos dados nas 3 condições de operação testadas. As campanhas de amostragem de gases seguiram as orientações estabelecidas nas normas técnicas NBR 10700, NBR 10701, NBR 10702, NBR 11966, NBR 11967, NBR 12019, NBR 12020, NBR 12021 e NBR 12827 da Associação Brasileira de Normas Técnicas, além dos métodos 1, 2, 3, 4, 5, 7, 13B, 18, 23, 26, 29 e 30 da Agência de Proteção Ambiental americana (Environmental Protection Agency-EPA). Selecionou-se o teste de Kruskal-Wallis (Estatística H), para comparação entre as medianas, ao nível de significância de 5\%.

Ao final do experimento avaliou-se a qualidade da solução alcalina de lavagem de gases através da análise dos parâmetros, pH, DBO, DQO, sólidos em suspensão, sólidos sedimentáveis, óleos e graxas, amônia, Agentes Tensoativos Aniônicos (ATA), benzeno, tolueno, etil benzeno, o-xileno, m,p-xilenos, chumbo total, cianetos, cobre total, cromo total, ferro solúvel, fluoreto total, índice de fenóis, mercúrio total, níquel total, sulfatos, sulfeto total e zinco. As metodologias utilizadas nas análises foram baseadas no Standard Methods for the examination of water and wastewater (APHA, 2005).

As características do produto carbonizado obtido (biochar) foram avaliadas quanto ao poder calorífico inferior (PCI), de acordo com o método ASTM-240-87, ensaios para avaliação dos extratos lixiviado e solubilizado, em conformidade com as metodologias estabelecidas nas normas técnicas NBR 10005 e NBR 10006 da Associação Brasileira de Normas Técnicas, além dos metais na massa bruta $(\mathrm{Pb}, \mathrm{V}, \mathrm{Zn}, \mathrm{Ba}, \mathrm{Be}$, $\mathrm{Cu}, \mathrm{Cr}, \mathrm{Li}, \mathrm{Mn}, \mathrm{Mo}, \mathrm{Sn}, \mathrm{Hg}$, Ta, Co, Ni, Te, Sb), em conformidade com os métodos SMWW 3120B, USEPA 6010 e SMWW 3111, e bifenilaspolicloradas (PCBs) pelo método EPA 8270D.

\section{RESULTADOS E DISCUSSÃO}

O aparato experimental operou no período sem registro de ocorrências de acidente ou interrupções, sem perda de controle de processo 
ou qualquer incidente que merecesse destaque. As avaliações relativas aos tempos e temperaturas de processo, rendimentos gravimétricos, consumos específicos de combustível auxiliar (cavacos de madeira) e produtividades horárias, encontram-se discriminadas na Tabela 2.

O reator foi operado em todos os ciclos na capacidade máxima de volume projetada $(3.000 \mathrm{~L})$, sendo que a massa de resíduos processada em cada ciclo, variou de acordo com a densidade dos materiais encaminhados ao aparato experimental. A massa mínima processada em um ciclo foi de $154,00 \mathrm{~kg}$ e a maior massa registrada foi de $401,70 \mathrm{~kg}$, demonstrando dessa forma a grande variação na densidade dos materiais processados. A média calculada da massa de carregamento dos resíduos nos 14 ciclos de processamento foi de $288,19 \mathrm{~kg}$, totalizando $4.034,70 \mathrm{~kg}$ de RSS ao final dos experimentos. O consumo específico de combustível auxiliar (cavacos de madeira) apresentou uma redução à medida quese verificou um aumento da produtividade horária da planta, conforme pode ser observado na Figura 2.

Para monitoramento das emissões atmosféricas foram estabelecidas três condições de operação para a verificação dos diversos parâmetros e os resultados de vazão, vazão normal, $\mathrm{MP}$ e $\mathrm{NO}_{\mathrm{x}}$, que encontram-se discriminados na Tabela 3.Aplicando-se o teste de Kruskal-Wallis (Estatística $\mathrm{H}$ ), para comparação entre as medianas, foram verificadas diferenças ao nível de significância de 5\% em relação às vazões, vazões normais $\left(\mathrm{H}_{\text {calc }}=7,20>\mathrm{H}_{0,05 ; 3 ; 3 ; 3}=5,60\right)$ e concentrações de óxidos de nitrogênio $\left(\mathrm{H}_{\text {calc }}=8,77>\mathrm{H}_{0,05 ; ; 4 ; 4}=5,68\right)$ nas 3 condições testadas. No entanto, não são verificadas diferenças significativas entre as concentrações de material particulado $\left(\mathrm{H}_{\text {calc }}=3,29<\mathrm{H}_{0,05 ; 3 ; 3 ; 3}=5,60\right)$, possivelmente em função do sistema de controle por via úmida instalado.

As medições relativas às concentrações de $\mathrm{SO}_{2}, \mathrm{HCl}, \mathrm{HF}$, Fluoretos, $\mathrm{Cl}_{2}$, além de dioxinas e furanos encontram-se representadas na Tabela 4, nas 3 condições de operação testadas.

Pode-se verificar, na Tabela 4, a redução expressiva das emissões de dioxinas e furanos na condição de operação 3, possivelmente em função de um melhor equilíbrio na combustão, além da condição 3 operar com maior volume de ar em excesso na fornalha. Outro fator a ser observado na redução das concentrações de dioxinas e furanos, diz respeito à interferência realizada na planta quanto ao local de injeção do gás de pirólise. O gás de pirólise na condição 1 foi injetado diretamente na fornalha de pós queima, entre os dois combustores de biomassa. A partir da condição 2, o gás passou a ser injetado em conjunto com o fluxo de ar de alimentação da combustão dos cavacos de madeira, proporcionando dessa forma maior turbulência, com o consequente favorecimento das condições de decomposição térmica das substâncias orgânicas no processo de combustão. As medições realizadas para verificação da concentração de metais nos gases encontram-se discriminadas na Tabela 5, nas três condições de operação do aparato experimental.

As concentrações dos outros metais apresentaram-se abaixo dos limites de quantificação dos métodos empregados para $\mathrm{Hg}\left(<0,001 \mathrm{mg} \cdot \mathrm{Nm}^{-3}\right)$,
Tabela 2 - Médias das perdas de massa verificadas nos ciclos de processamento de Resíduos de Serviços de Saúde.

\begin{tabular}{l|c|c|c}
$\begin{array}{l}\text { Quantidade } \\
\text { de ciclos }\end{array}$ & $\begin{array}{c}\text { Tempo de } \\
\text { processo }(\mathrm{h})\end{array}$ & $\begin{array}{c}\text { Temperatura do fluido } \\
\text { de processo }\left({ }^{\circ} \mathrm{C}\right)\end{array}$ & $\begin{array}{c}\text { Perda de massa } \\
(\%)\end{array}$ \\
\hline 1 & 2 & $329^{*}$ & $46,75^{*}$ \\
\hline 8 & 3 & $325^{*}$ & $58,51^{*}$ \\
\hline 4 & 4 & $325^{\star}$ & $58,77^{*}$ \\
\hline 1 & 6 & $325^{\star}$ & $56,67^{\star}$ \\
\hline
\end{tabular}

*Valores médios.

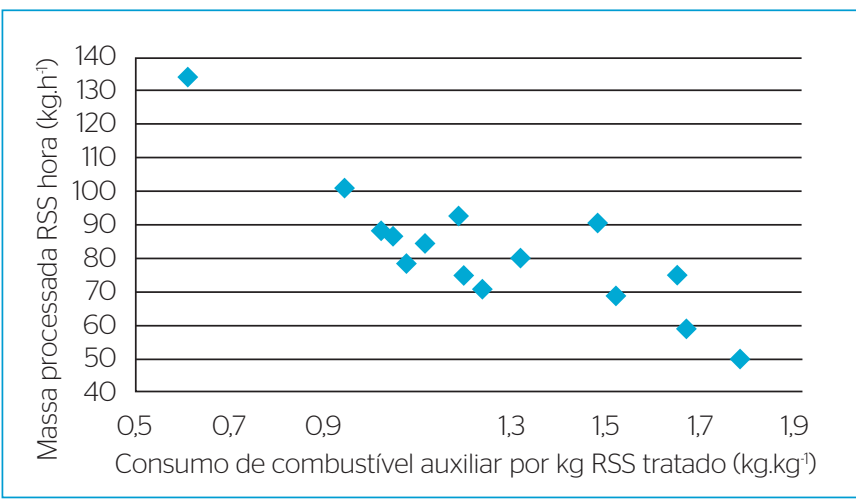

RSS: Resíduos de Serviços de Saúde.

Figura 2 - Massa processada de Resíduos de Serviços de Saúde em relação ao consumo específico de combustível.

Tabela 3 - Medições de vazão dos gases, concentração de material particulado e óxidos de nitrogênio nos lançamentos.

\begin{tabular}{|c|c|c|c|c|}
\hline $\begin{array}{l}\text { Condiçãao de } \\
\text { operação }\end{array}$ & $\begin{array}{l}\text { Vazão } \\
\left(\mathrm{m}^{3} \cdot \mathrm{h}^{-1}\right)\end{array}$ & $\begin{array}{c}\text { Vazão Normal } \\
\left(\mathrm{Nm}^{3} / \mathrm{h}^{-1}\right)\end{array}$ & $\begin{array}{c}{\left[\mathrm{MP}^{*}\right.} \\
\left(\mathrm{mg} \cdot \mathrm{Nm}^{-3}\right)\end{array}$ & $\begin{array}{c}{\left[\mathrm{NO}_{\mathrm{x}}\right]^{*}} \\
\left(\mathrm{mg} \cdot \mathrm{Nm}^{-3}\right)\end{array}$ \\
\hline \multirow{3}{*}{1} & 1058,66 & 799,75 & 49,34 & 40,15 \\
\hline & 1081,60 & 799,12 & 53,76 & 104,28 \\
\hline & 1080,32 & 799,19 & 29,49 & 23,07 \\
\hline \multirow{3}{*}{2} & 2597,25 & 2017,05 & 84,78 & 344,56 \\
\hline & 2595,67 & 1976,96 & 100,91 & 160,97 \\
\hline & 2583,54 & 1998,04 & 31,29 & 240,34 \\
\hline \multirow{3}{*}{3} & 3043,76 & 2285,77 & 63,43 & 114,3 \\
\hline & 3016,81 & 2358,48 & 61,08 & 78,54 \\
\hline & 3017,78 & 2347,58 & 57,84 & 80,89 \\
\hline
\end{tabular}

Obs: Para avaliação de $\mathrm{NO}_{x}$ foram realizadas quatro medidas; ${ }^{V}$ Valores das concentrações corrigidas para a base $7 \%$ de $\mathrm{O}_{2}$

$\mathrm{Ti}\left(<0,010 \mathrm{mg} \cdot \mathrm{Nm}^{-3}\right), \mathrm{As}\left(<0,010 \mathrm{mg} \cdot \mathrm{Nm}^{-3}\right)$, Co e $\mathrm{Ni}\left(<0,098 \mathrm{mg} \cdot \mathrm{Nm}^{-3}\right)$, Se $\left(<0,010 \mathrm{mg} \cdot \mathrm{Nm}^{-3}\right)$, Te $\left(<0,010 \mathrm{mg} \cdot \mathrm{Nm}^{-3}\right)$, Sb $\left(<0,005 \mathrm{mg} \cdot \mathrm{Nm}^{-3}\right)$, $\mathrm{Cr}\left(<0,049 \mathrm{mg} \cdot \mathrm{Nm}^{-3}\right), \operatorname{Pt}\left(<0,010 \mathrm{mg} \cdot \mathrm{Nm}^{-3}\right), \operatorname{Pd}\left(<0,010 \mathrm{mg}^{-N_{m}}{ }^{-3}\right), \operatorname{Rh}(<0,010$ mg.Nm $\left.{ }^{-3}\right), \mathrm{V}\left(<0,010 \mathrm{mg} \cdot \mathrm{Nm}^{-3}\right)$ e Sn $\left(<0,010 \mathrm{mg} \cdot \mathrm{Nm}^{-3}\right)$. A não quantificação da maior parte dos metais no fluxo gasoso dos lançamentos, bem como as baixas concentrações verificadas nos lançamentos de manganês, cobre, chumbo e cádmio, podem indicar uma tendência dos elementos metálicos de permanecer no reator de pirólise, em mistura com o produto carbonizado. Esse fato pode ser evidenciado com a verificação das baixas concentrações de metais nos resultados das análises da solução alcalina de lavagem de gases, comparando-se com as concentrações de compostos orgânicos aromáticos (Benzeno, Tolueno, Etilbenzeno, m,p-xilenos, o-xileno e índice de fenóis) e amônia, conforme resultados apresentados 
Tabela 4 - Concentração das substâncias $\mathrm{SO}_{2}, \mathrm{HCl}, \mathrm{HBr}, \mathrm{HF}$, Fluoretos, $\mathrm{Cl}_{2}$, Dioxinas e Furanos nas 3 condições de operação.

\begin{tabular}{|c|c|c|c|c|c|c|c|}
\hline Condição & $\begin{array}{c}{\left[\mathrm{SO}_{2}\right]^{*}} \\
\left(\mathrm{mg} \cdot \mathrm{Nm}^{-3}\right)\end{array}$ & $\begin{array}{c}{[\mathrm{HCl}]^{*}} \\
\left(\mathrm{mg} \cdot \mathrm{Nm}^{-3}\right)\end{array}$ & $\begin{array}{c}{[\mathrm{HBr}]^{*}} \\
\left(\mathrm{mg} \cdot \mathrm{Nm}^{-3}\right)\end{array}$ & $\begin{array}{c}\text { [HF] }^{*} \\
\left(\mathrm{mg} \cdot \mathrm{Nm}^{-3}\right)\end{array}$ & $\begin{array}{c}\text { [Fluoretos] }^{*} \\
\left(\mathrm{mg} \cdot \mathrm{Nm}^{-3}\right)\end{array}$ & $\begin{array}{c}{\left[\mathrm{Cl}_{2}\right]^{*}} \\
\left(\mathrm{mg} \cdot \mathrm{Nm}^{-3}\right)\end{array}$ & $\begin{array}{l}\text { Dioxinas e } \\
\text { Furanos* } \\
\left(\eta g . \mathrm{Nm}^{-3}\right)\end{array}$ \\
\hline \multirow{3}{*}{1} & $<1,595$ & 2,150 & $<0,091$ & $<0,134$ & 0,110 & $<0,183$ & \multirow{3}{*}{0,6371} \\
\hline & 4,560 & $<0,009$ & $<0,091$ & $<0,134$ & 1,100 & $<0,183$ & \\
\hline & $<1,595$ & 0,030 & $<0,091$ & $<0,134$ & 0,300 & $<0,183$ & \\
\hline \multirow{3}{*}{2} & 1,940 & $<0,009$ & $<0,092$ & $<0,125$ & 0,150 & $<0,183$ & \multirow{3}{*}{ 0,1095 } \\
\hline & $<1,592$ & $<0,009$ & $<0,092$ & $<0,125$ & 0,070 & $<0,183$ & \\
\hline & 5,490 & $<0,009$ & $<0,092$ & 0,200 & 0,260 & $<0,183$ & \\
\hline \multirow{3}{*}{3} & 7,190 & $<0,009$ & $<0,092$ & 3,100 & $<0,012$ & $<0,184$ & \multirow{3}{*}{0,0162} \\
\hline & 3,610 & $<0,009$ & $<0,092$ & 2,750 & $<0,012$ & $<0,184$ & \\
\hline & 7,230 & $<0,009$ & $<0,092$ & 2,750 & $<0,012$ & $<0,184$ & \\
\hline
\end{tabular}

*Valores das concentrações corrigidas para a base $7 \%$ de $\mathrm{O}_{2}$

na Tabela 6. O valor obtido para concentração de sulfatos de 131,30 mg.. $\mathrm{L}^{-1}$, pode estar relacionado com a presença de substâncias surfactantes, comumente utilizadas como agentes sanitizantes nos estabelecimentos de saúde. Entretanto, o valor obtido de $0,45 \mathrm{mg} \cdot \mathrm{L}^{-1}$, para o parâmetro ATA, não permite afirmar que o sulfato presente na solução alcalina é proveniente desse tipo de agente sanitizante, apesar desses compostos possivelmente já se encontrarem degradados após a volatilização e absorção pelo meio líquido.

A avaliação da concentração da carga orgânica presente na solução alcalina pode ser expressa principalmente pelos resultados obtidos nos parâmetros DBO e DQO. As concentrações representativas de substâncias orgânicas retidas na fase líquida representamuma perda de energia para o sistema. Caso o fluxo da fase orgânica volatilizada no reator de pirólise fosse direcionado à fornalha de pós queima, sem a passagem pela via úmida, o requisito de combustível auxiliar seria reduzido. No entanto, a ação do sistema via úmida no controle de elementos precursores de poluentes perigosos pode proporcionar uma operação com maior segurança ambiental. Essa observação pode ser evidenciada através da concentração de fluoreto total na solução alcalina.

Levando-se em consideração os limites de referência estabelecidos pela série de Normas Técnicas Brasileiras de classificação de resíduos (ABNT, 2004), todos os resultados obtidos para as concentrações de contaminantes encontram-se abaixo dos limites no extrato lixiviado, como pode ser observado na Tabela 7 .

A Tabela 8 apresenta os resultados das concentrações de Al, As, $\mathrm{Ba}, \mathrm{Cd}, \mathrm{Pb}$, cianeto, cloreto, Fe, fluoreto, $\mathrm{Mn}, \mathrm{Hg}$, nitrato, $\mathrm{Ag}, \mathrm{Se}, \mathrm{Na}$, sulfato, surfactantes, $\mathrm{Zn}, \mathrm{Cu}, \mathrm{Cr}$ e fenóis totais no extrato solubilizado.

O parâmetro cloreto encontra-se acima do limite de referência da norma, evidenciando a tendência de alguns compostos clorados permanecerem no produto carbonizado. Outro parâmetro que se apresenta acima dos limites, fenóis totais, evidencia a tendência de formação de produtos aromáticos durante as reações de decomposição de compostos orgânicos na atmosfera redutora do reator de pirólise. As concentrações de surfactantes apresentaram-se acima dos limites de referência, indicando que essas substâncias encontram-se presentes em quantidades razoáveis nos resíduos dos estabelecimentos de
Tabela 5 - Concentração de metais nos gases de exaustão.

\begin{tabular}{|c|c|c|c|c|}
\hline $\begin{array}{l}\text { Condição de } \\
\text { operação }\end{array}$ & $\begin{array}{c}{[\mathrm{Mn}]^{*}} \\
\left(\mathrm{mg} \cdot \mathrm{Nm}^{-3}\right)\end{array}$ & $\begin{array}{c}{[\mathrm{Cu}]^{*}} \\
\left(\mathrm{mg} \cdot \mathrm{Nm}^{-3}\right)\end{array}$ & $\begin{array}{c}{[\mathrm{Pb}]^{*}} \\
\left(\mathrm{mg} \cdot \mathrm{Nm}^{-3}\right)\end{array}$ & $\begin{array}{c}{[\mathrm{Cd}]^{*}} \\
\left(\mathrm{mg} \cdot \mathrm{Nm}^{-3}\right)\end{array}$ \\
\hline \multirow{3}{*}{1} & 0,142 & $<0,098$ & $<0,098$ & $<0,001$ \\
\hline & 0,140 & $<0,098$ & $<0,098$ & $<0,001$ \\
\hline & 0,132 & $<0,098$ & $<0,098$ & $<0,001$ \\
\hline \multirow{3}{*}{2} & 0,232 & $<0,098$ & $<0,098$ & $<0,001$ \\
\hline & 0,208 & 0,110 & $<0,098$ & 0,011 \\
\hline & 0,453 & $<0,098$ & $<0,098$ & $<0,001$ \\
\hline \multirow{3}{*}{3} & 0,118 & $<0,096$ & $<0,096$ & $<0,001$ \\
\hline & 0,089 & $<0,096$ & $<0,096$ & $<0,001$ \\
\hline & 0,116 & $<0,096$ & $<0,096$ & $<0,001$ \\
\hline
\end{tabular}

*Valores das concentrações corrigidas para a base 7\% de $\mathrm{O}_{2}$.

saúde, possivelmente por fazerem parte da composição de agentes sanitizantes utilizados.

Em razão das concentrações dos parâmetros cloretos, fenóis totais, ferro, manganês e surfactantes encontrarem-se acima dos limites de referência listados na norma técnica NBR 10.006(ABNT,2004), o resíduo deve ser enquadrado como classe IIA, de acordo com os critérios da norma técnica NBR 10.004 (ABNT, 2004).O valor obtido de 5.692 kcal.kg-1 na avaliação do PCI do produto carbonizado evidencia a vocação desse material para ser utilizado como combustível em processos térmicos. Entretanto, a presença de contaminantes avaliados no extrato lixiviado e no extrato solubilizado, bem como na massa bruta do resíduo, sugerem que serão necessários cuidados especiais para a utilização desse produto em processos de combustão, considerando-se o risco de emissões de produtos perigosos. A Tabela 9 apresenta os resultados das concentrações de alguns metais e semi-metais relevantes, além de cloretos e PCBs na massa bruta do produto carbonizado.

Dentre os metais avaliados, os resultados mais expressivos foram encontrados para as concentrações de zinco e manganês. Além desses metais, apenas chumbo, cobre e níquel apresentaram-se acima dos limites de quantificação dos métodos empregados. Não se verificou concentração de PCBs acima dos limites de detecção do método empregado para avaliação desse parâmetro. 
Tabela 6 - Concentrações de contaminantes na solução de lavagem de gases após os 14 ciclos de tratamento de Resíduos de Serviços de Saúde.

\begin{tabular}{|c|c|c|}
\hline Descrição & Unid. & Valores \\
\hline Amônia & $\mathrm{mgNH}_{3} \mathrm{~L}^{-1}$ & 2,11 \\
\hline ATA & $\mathrm{mg} \cdot \mathrm{L}^{-1}$ & 0,45 \\
\hline Benzeno & g. $L^{-1}$ & 3,94 \\
\hline Tolueno & g. $\mathrm{L}^{-1}$ & 36,1 \\
\hline Etilbenzeno & g. $L^{-1}$ & 23,9 \\
\hline O-Xileno & g. $L^{-1}$ & 1,31 \\
\hline m,p-xilenos & g. $L^{-1}$ & 19,3 \\
\hline Chumbo total & $m g . L^{-1}$ & 0,01 \\
\hline Cianetos & mgCN:L-1 & $<0,01$ \\
\hline Cobre total & $m g . L^{-1}$ & 0,07 \\
\hline Cromo total & $\mathrm{mg} \cdot \mathrm{L}^{-1}$ & $<0,05$ \\
\hline DBO & $\mathrm{mgO}_{2} \mathrm{~L}^{-1}$ & 540 \\
\hline DQO & $\mathrm{mgO}_{2} \mathrm{~L}^{-1}$ & 1348 \\
\hline Ferro solúvel & $\mathrm{mg} \cdot \mathrm{L}^{-1}$ & 0,85 \\
\hline Fluoreto total & $m g \cdot L^{-1}$ & 1,11 \\
\hline Índice de fenóis & $m g \cdot L^{-1}$ & 0,17 \\
\hline Mercúrio total & $m g \cdot L^{-1}$ & $<1$ \\
\hline Níquel total & $m g . L^{-1}$ & 0,03 \\
\hline Óleos e graxas & $m g \cdot L^{-1}$ & 13 \\
\hline $\mathrm{pH}$ & - & 9,3 \\
\hline Sólidos em suspensão & $m g . L^{-1}$ & $<4$ \\
\hline Sólidos sedimentáveis & $m g \cdot L^{-1}$ & $<0,01$ \\
\hline Sulfatos & $\mathrm{mgSO}_{4}^{2} \cdot \mathrm{L}^{-1}$ & 131,3 \\
\hline Sulfeto total & $\mathrm{mgS}^{2} \mathrm{~L}^{-1}$ & $<0,1$ \\
\hline Zinco & $m g \cdot L^{-1}$ & 0,14 \\
\hline
\end{tabular}

Tabela 7 - Concentrações de Arsênio, Bário, Cádmio, Chumbo, Cromo total, Fluoreto, Mercúrio, Prata e Selênio no extrato lixiviado.

\begin{tabular}{|c|c|c|c|}
\hline Parâmetro & Unidade & Resultados & $\begin{array}{l}\text { Limite de refe- } \\
\text { rência* }\end{array}$ \\
\hline Arsênio & mg As. $L^{-1}$ & $<0,01$ & 1,0 \\
\hline Bário & mg Ba. $\mathrm{L}^{-1}$ & $<0,10$ & 70,0 \\
\hline Cádmio & $\mathrm{mg} \mathrm{Cd.L^{-1 }}$ & $<0,01$ & 0,5 \\
\hline Chumbo & mg Pb. $\mathrm{L}^{-1}$ & $<0,1$ & 1,0 \\
\hline Cromo total & $\mathrm{mg} \mathrm{Cr} \cdot \mathrm{L}^{-1}$ & $<0,05$ & 5,0 \\
\hline Fluoreto & $m g$ F: $L^{-1}$ & $<0,48$ & 15,0 \\
\hline Mercúrio & $\mathrm{mg} \mathrm{Hg.L^{-1 }}$ & $<0,001$ & 0,1 \\
\hline Prata & $\mathrm{mg} \mathrm{Ag}^{-1}$ & $<0,05$ & 5,0 \\
\hline Selênio & mg Se. $L^{-1}$ & $<0,01$ & 1,0 \\
\hline
\end{tabular}

*Limite máximo no extrato obtido no ensaio de lixiviação (NBR 10.005: ABNT, 2004).

\section{CONCLUSÕES}

O processo "Pyrolix" demonstrou ser uma opção promissora para ser adotada como alternativa no gerenciamento dos RSS, por possibilitar o autossuprimento do combustível auxiliar através do beneficiamento dos resíduos de poda, verificado o consumo específico obtido na melhor condição de operação de $0,61 \mathrm{~kg}$ de cavacos de madeira por kg de RSS processado.

O processo avaliado apresenta uma série de vantagens verificadas no gerenciamento de resíduos, tais como redução de massa entre 46,75\% e 58,77\%, utilização de combustível auxiliar de baixo custo (biomassa) na planta de tratamento, eliminação de lixiviados na disposição final,
Tabela 8 - Concentrações de $\mathrm{Al}, \mathrm{As}, \mathrm{Ba}, \mathrm{Cd}, \mathrm{Pb}$, cianeto, cloreto, $\mathrm{Cu}$ e $\mathrm{Cr}$ totais, fenóis totais, $\mathrm{Fe}$, fluoreto, $\mathrm{Mn}, \mathrm{Hg}$, nitrato, $\mathrm{Ag}$, Se, $\mathrm{Na}$, sulfato, surfactantes e Zn no extrato solubilizado.

\begin{tabular}{|c|c|c|c|c|}
\hline Parâmetro & Unidade & \multicolumn{2}{|c|}{ Resultados } & $\begin{array}{l}\text { Limite de } \\
\text { referência* }\end{array}$ \\
\hline Alumínio & mg Al..-1 & $<0,100$ & $<0,100$ & 0,200 \\
\hline Arsênio & $\mathrm{mg} A s . L^{-1}$ & $<0,010$ & $<0,010$ & 0,010 \\
\hline Bário & mg Ba. $\mathrm{L}^{-1}$ & 0,070 & 0,070 & 0,700 \\
\hline Cádmio & mg Cd..-1 & $<0,001$ & $<0,001$ & 0,005 \\
\hline Chumbo & $\mathrm{mg} \mathrm{Pb} . \mathrm{L}^{-1}$ & $<0,010$ & $<0,010$ & 0,010 \\
\hline Cianeto & mg CN:L-1 & $<0,010$ & $<0,010$ & 0,070 \\
\hline Cloreto & mg Cl:.-1 & 449,070 & 451,820 & 250,000 \\
\hline Cobre total & mg Cu. $\mathrm{L}^{-1}$ & $<0,050$ & $<0,050$ & 2,000 \\
\hline Cromo total & $\mathrm{mg} \mathrm{Cr.L^{-1 }}$ & $<0,050$ & $<0,050$ & 0,050 \\
\hline Fenóis totais & $\mathrm{mg} \cdot \mathrm{L}^{-1}$ & 0,430 & 0,430 & 0,010 \\
\hline Ferro & mg Fe. $L^{-1}$ & 1,210 & 1,450 & 0,300 \\
\hline Fluoreto & $m g ~ F: L^{-1}$ & 0,830 & 0,820 & 1,500 \\
\hline Manganês & $\mathrm{mg} \mathrm{Mn.L^{-1 }}$ & 0,980 & 1,280 & 0,100 \\
\hline Mercúrio & $\mathrm{mg} \mathrm{Hg} \cdot \mathrm{L}^{-1}$ & $<0,001$ & $<0,001$ & 0,001 \\
\hline Nitrato & $m g ~ N . L^{-1}$ & $<1,000$ & $<1,000$ & 10,000 \\
\hline Prata & $\mathrm{mg} \mathrm{Ag} \cdot \mathrm{L}^{-1}$ & $<0,050$ & $<0,050$ & 0,050 \\
\hline Selênio & mg Se. $L^{-1}$ & $<0,010$ & $<0,010$ & 0,010 \\
\hline Sódio & mg Na. $\mathrm{L}^{-1}$ & 155,550 & 157,250 & 200,000 \\
\hline Sulfato & $\mathrm{mg} \mathrm{SO}_{4}{ }^{2-L^{-1}}$ & 133,700 & 144,300 & 250,000 \\
\hline Surfactantes & $\mathrm{mg} \cdot \mathrm{L}^{-1}$ & 1,190 & 1,190 & 0,500 \\
\hline Zinco & $\operatorname{mg} Z n L^{-1}$ & 0,330 & 0,310 & 5,000 \\
\hline
\end{tabular}

*Limite máximo no extrato obtido no ensaio de solubilização (NBR 10.006:ABNT,2004).

Tabela 9 - Concentrações de alguns metais e semi-metais relevantes, além de cloretos e PCBs no produto carbonizado.

\begin{tabular}{|c|c|c|}
\hline Descrição & Unidade & Valores \\
\hline Chumbo & mg.kg ${ }^{-1}$ & 44,56 \\
\hline Vanádio & mg.kg ${ }^{-1}$ & $<1,00$ \\
\hline Zinco & mg.kg ${ }^{-1}$ & 804,50 \\
\hline Bário & mg.kg-1 & $<1,00$ \\
\hline Berílio & mg.kg-1 & $<1,00$ \\
\hline Cobre & mg.kg ${ }^{-1}$ & 17,02 \\
\hline Lítio & mg.kg ${ }^{-1}$ & $<1,00$ \\
\hline Manganês & mg.kg ${ }^{-1}$ & 99,62 \\
\hline Molibdênio & mg.kg-1 & $<1,00$ \\
\hline Estanho & mg.kg ${ }^{-1}$ & $<1,00$ \\
\hline Cromo & mg.kg ${ }^{-1}$ & $<5,00$ \\
\hline Mercúrio & mg.kg-1 & $<0,05$ \\
\hline Tálio & mg.kg ${ }^{-1}$ & $<1,00$ \\
\hline Cobalto & mg.kg ${ }^{-1}$ & $<5,00$ \\
\hline Níquel & mg.kg-1 & 5,01 \\
\hline Telúrio & mg.kg ${ }^{-1}$ & $<5,00$ \\
\hline Antimônio & mg.kg-1 & $<0,50$ \\
\hline Cloretos & mg.kg ${ }^{-1}$ & 1801,79 \\
\hline PCBs & mg.kg ${ }^{-1}$ & $<0,31$ \\
\hline
\end{tabular}


além da possibilidade de encaminhamento do produto carbonizado para a disposição em um aterro sanitário convencional. $\mathrm{O}$ aparato testado demonstrou melhor segurança ambiental na terceira condição de operação testada, em relação às emissões de contaminantes para a atmosfera, levando-se em consideração o valor obtido na concentração de dioxinas e furanos de $0,0162 \mathrm{ng} \cdot \mathrm{Nm}^{-3}$.

O produto carbonizado obtido no processo, apesar da possibilidade de aplicação energética em outros processos térmicos, em função do PCI de 5.692,0 kcal.kg-1 , pode apresentar limitações decorrentes da presença de contaminantes no produto, verificada a concentração de cloreto de 1801,79 mg.kg-1.

A solução proposta para o município de Belo Horizonte apresentou viabilidade técnica e ambiental, de acordo com os resultados avaliados, com potencial para ser aplicada em outros municípios no Brasil e no mundo, que enfrentam o gerenciamento adequado dos RSS como um desafio a ser transposto pelo poder público municipal.

\section{AGRADECIMENTOS}

Os autores manifestam seus agradecimentos à Superintendência de Limpeza Urbana (SLU) da Prefeitura Municipal de Belo Horizonte e àFundação de Amparo à Pesquisa do Estado de Minas Gerais (FAPEMIG), pelo financiamento das pesquisas e ao Conselho Nacional de Desenvolvimento Científico e Tecnológico (CNPq) e à Coordenação de Aperfeiçoamento de Pessoal de Nível Superior (CAPES), pelo apoio institucional.

\section{REFERÊNCIAS}

ALVIM-FERRAZ, M.C.M. \& AFONSO, S.A.V. Incineration of different types of medical wastes: emission factors for gaseous emissions. Atmospheric Environment, v. 37, p. 5415-5422, 2003.

ANVISA - Agência Nacional de Vigilância Sanitária. Gerenciamento dos Resíduos de Serviço de Saúde. 2006. Disponível em: <http://www. anvisa.gov.br/servicosaude/manuais/manual_gerenciamento_residuos. pdf.> Acesso em 04 de abril 2011

APHA; AWWA; WEF.Standard methods for the examination of water and wastewater.21. ed. Washington: APHA, 2005

ASSOCIAÇÃO BRASILEIRA DE NORMAS TÉCNICAS. NBR 10004, NBR 10005, NBR 10006, NBR 10007. Resíduos sólidos - Coletânea de Normas. $2^{\mathrm{a}}$ ed. Rio de Janeiro, 2004. 124 p.

AVENELL, C.S.; SAINZ-DIAZ, C.I.; GRIFFITHS, A.J. Solid waste pyrolysis in a pilot-scale batch pyrolyser. Fuel, v. 75, p. 1167-1174, 1996

BEESLEY, L.; MORENO-JIMÉNEZ, E; GOMEZ-EYLES, J. L.; HARRIS, E.; ROBINSON, B. SIZMUR, T. A review of biochars' potential role in the remediation, revegetation and restoration of contaminated soils. Environmental Pollution, v. 159, p. 3269-3282, 2011.

BRANDRUP, J. Recycling and Recovery of Plastics. New York (EUA): Hanser/Gardner Publications, 1996.

CABALLERO, J.A., FONT, R., ESPERANZA, M.M. Kinetics of the thermal decomposition oftannery waste.Journal of Analytical and Applied Pyrolysis, v. 46, p. 165-181, 1998.

CONAMA - Conselho nacional do meio ambiente. Resolução no. 358 de 29 de abr. de 2005. Dispõe sobre o tratamento e a disposição final dos resíduos dos serviços de saúde e dá outras providências. Disponível em: $\quad$ <http://www.mma.gov.br/port/conama/res/res05/res35805.pdf.> Acesso em 20 de outubro 2010
DEBELA, F.; THRING, R.W.; AROCENA, J.M. Immobilization of Heavy Metals by Co-Pyrolysis of Contaminated Soil With Woody Biomass. Water Air Soil Pollut, v. 223, p. 1161-1170, 2012.

DENG N.A.; YUFENG, Z:; JUHONG, L.; CHANGZHONG, X. A new pyrolysis technology and equipment for treatment of municipal household garbage and hospital waste. Renewable Energy, v. 28, p. 2383-2393, 2003

HUFFMAN, G.L. \& LEE, C.C. Medical waste management/incineration. Journal of Hazardous Materials, v. 48, p.1-30, 1996.

MALKOW, T. Novel and innovative pyrolysis and gasification technologies for energy efficient and environmentally sound MSW disposal. Waste Management, v. 24, p. 53-79, 2004.

MELO, G.C.B.; TORRES FILHO, A.; BORGES, M.E.; BICALHO, P.A.; VALENTE, V.B. Avaliação de desempenho de um reator de pirólise no tratamento de uma amostra simulada de resíduos de serviço de saúde. In: SEMINÁRIO NACIONAL DE RESÍDUOS SÓLIDOS, 4., 2008 , Palmas. Anais... Palmas: 2008 .p. 1-8.

PESQUISA NACIONAL DE SANEAMENTO BÁSICO (PNSB, 2008). Rio de Janeiro: 2010. ISBN: 978-85-240-4135-8. Disponível em: <http://www.ibge. gov.br/home/estatistica/populacao/condicaodevida/pnsb2008/ default. shtm>. Acesso em 23 de fevereiro de 2011.

SMITH, J.L.; COLLINS, P.H.; BAILEY, V.L. Soil Biology \& Biochemistry, v. 42, p. 2345-2347. 2010.

USEPA - United States Environmental Protection Agency. Technology Transfer Network. 2011. Disponível em: <http://www.epa.gov/ttn/>. Acesso em 20 de fevereiro de 2011

ZHU, H.M.; YAN, J.H.; JIANG, X.G.; LAI, Y.E.; CEN, K.F. Study on pyrolysis of typical medical waste materials by using TG-FTIR analysis. $R$ Journal of Hazardous Materials, v. 153, p. 670-676, 2008. 